\title{
La gencive péri-implantaire
}

\section{The peri-implant gingiva}

\section{MOTS-CLEFS : \\ - Gencive, implant, épithélium, tissu conjonctif \\ KEYWORDS: \\ - Gingiva, implant, epithelium, connective tissue}

\section{AOS 2013;264:4-8 \\ DOI: $10.1051 / \mathrm{aos} / 2013402$ (C) EDP Sciences 2013}

\section{Résumé}

Au début de l'implantologie dentaire, l'émergence transgingivale de l'implant a été un sujet important de controverses, certains voyant dans la brèche réalisée une porte d'entrée aux germes bactériens, d'autres assurant qu'il existait une étanchéité de cette structure. Cette controverse s'est depuis éteinte, les travaux les plus récents ayant montré l'équivalent d'une attache épithélio-conjonctive sur le titane, assurant une herméticité des tissus autour du pilier implantaire. Un rappel de ces notions semble cependant nécessaire afin d'appuyer sur des bases scientifiques souvent ignorées, et pourtant nécessaires, les traitements proposés pour les pathologies de ce sillon.

\section{Abstract}

\begin{abstract}
In the early days of dental implantology, the transgingival emergence of the implant was a major topic of controversy; some thought that the resulting breach provided a way in for bacterial organisms, whereas others were convinced that this structure was in fact sealed. This controversy has since died down, the most recent studies having revealed something equivalent to an epithelioconjunctive attorchment to the titanium, which ensures that the tissues are in fact sealed around the implanted pillar. However, it seems necessary to reiterate these ideas in order to establish a sound scientific basis, necessary but often overlooked, for the treatments proposed for disorders of this sulcus.
\end{abstract}

Dr Marc Bert
Docteur en Sciences odontologiques
Expert près la cour d'Appel de Paris

\section{MODĖLE BIOLOGIQUE : SILLON GINGIVO-DENTAIRE}

La gencive est constituée de deux tissus, l'épithélium et le tissu conjonctif, séparés par une membrane basale.

\section{Épithélium}

Lépithélium, tissu non vascularisé, se développe au contact du tissu conjonctif sous-jacent au niveau de sa couche initiale dite basale ou germinative où les cellules se divisent par mitose. Ces cellules migrent ensuite vers l'extérieur (couche épineuse, puis granuleuse) en perdant progressivement leur noyau et sont éliminées par desquamation au niveau de la couche superficielle.

Lépithélium externe est kératinisé, comprend de nombreuses couches cellulaires et présente des digitations à l'intérieur du tissu conjonctif afin d'en augmenter la surface de contact, donc la nutrition.

Lépithélium du sillon gingivo-dentaire montre un nombre réduit de couches cellulaires, la disparition des digitations et l'absence de kératine. Au niveau de la dent, cet épithélium ne comporte plus que quelques couches cellulaires qui viennent s'attacher au cément par l'intermédiaire de trois structures : des hémidesmosomes, une lame basale et une couche de mucopolysaccharides (ou protéoglycans), véritable colle cellulaire. 


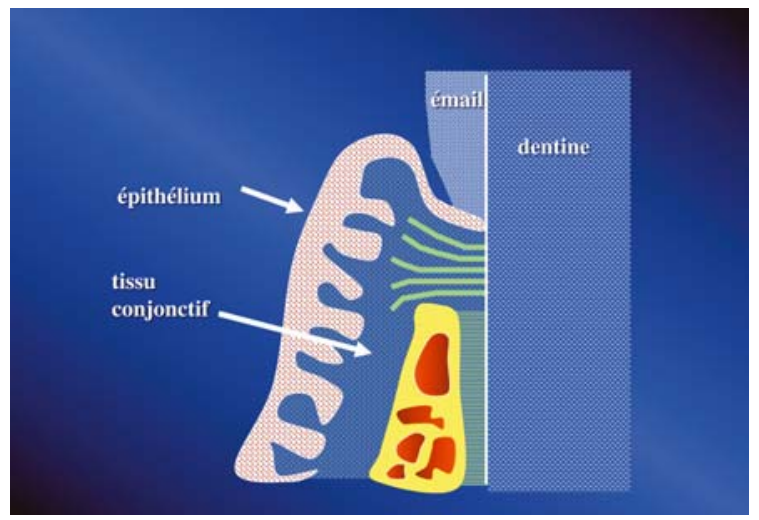

\section{Tissu conjonctif}

Le tissu conjonctif, vascularisé, comprend des cellules (ostéoblastes, fibroblastes, chondroblastes...), des fibres, principalement de collagène et une substance fondamentale, matériau de remplissage entre les cellules et les fibres qu'elle enrobe.

Au niveau du sillon gingivo-dentaire, le tissu conjonctif comprend des fibroblastes et des fibres de collagène avec:

I des fibres dento-gingivales qui vont du cément à la gencive;

I des fibres gingivo-périostées qui se tendent de la crête alvéolaire jusqu'au périoste vestibulaire et lingual ;

I des fibres alvéolo-gingivales qui vont de la crête alvéolaire à la gencive libre ;

I des fibres circulaires, semi-circulaires, intergingivales et trans-septales.

L'ensemble forme l'attache épithélio-conjonctive, composée d'un " collage » épithélial et d'un " ancrage » conjonctif (fig. 1).

Ses moyens de défense sont constitués par :

I lépithélium stratifié squameux de recouvrement,
4 Fig. 1:

L'attache épithélio-conjonctive dentaire.

Lépithélium externe est kératinisé et présente des digitations à l'intérieur du tissu conjonctif. Lépithélium interne n’est pas kératinisé et le nombre de couches cellulaires diminue vers la base du sillon pour n'avoir que 3 ou 4 couches au niveau de l'attache. Les fibres conjonctives sont ancrées dans le cément et perpendiculaires à sa surface. avec son potentiel régénérateur puissant et rapide qui entraîne dans sa desquamation les substances toxiques qui auraient pu le pénétrer ;

I la kératinisation qui oppose à la pénétration des germes une couche imperméable en surface ;

I la couche de mucopolysaccharide sécrétée par les cellules épithéliales qui réalise un véritable scellement de l'espace tissu-dent ;

I une couche de glycoprotéine qui recouvre les surfaces muqueuses et dentaires.

Cimasoni [1] a montré qu'il existait dans le sillon gingivo-dentaire une sécrétion permanente dans laquelle on retrouve des leucocytes polynucléaires qui migrent en permanence des vaisseaux dans le tissu conjonctif, puis dans le sillon gingivo-dentaire à travers ses quelques couches épithéliales et des macrophages qui jouent un rôle important dans la destruction, la phagocytose et lélimination des divers pathogènes. En cas d'inflammation, à cette sécrétion d'origine interstitielle s'ajoute une extravasion sérique liée à la dilatation des capillaires (fig. 2 A et B). Depuis Oliver [2], de nombreux auteurs ont montré que l'augmentation de la sécrétion de fluide créviculaire est directement corrélée à l'augmentation de l'inflammation (fig. 3).
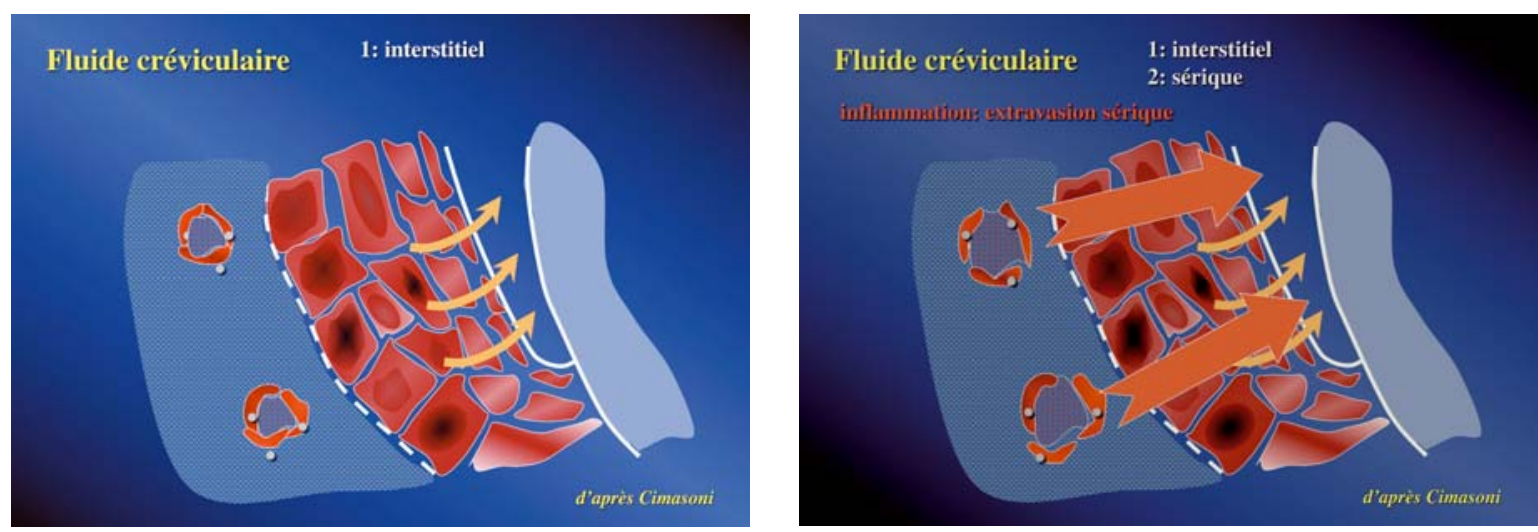

$\triangle$ Fig. 2A, 2B :

Il existe dans le sillon gingivo-dentaire sain une sécrétion permanente de liquide interstitiel. En cas d'inflammation, la dilatation des capillaires entraîne une extravasion sérique qui s'ajoute à la sécrétion interstitielle et augmente la quantité de fluide créviculaire (d'après Cimasoni [1]). 


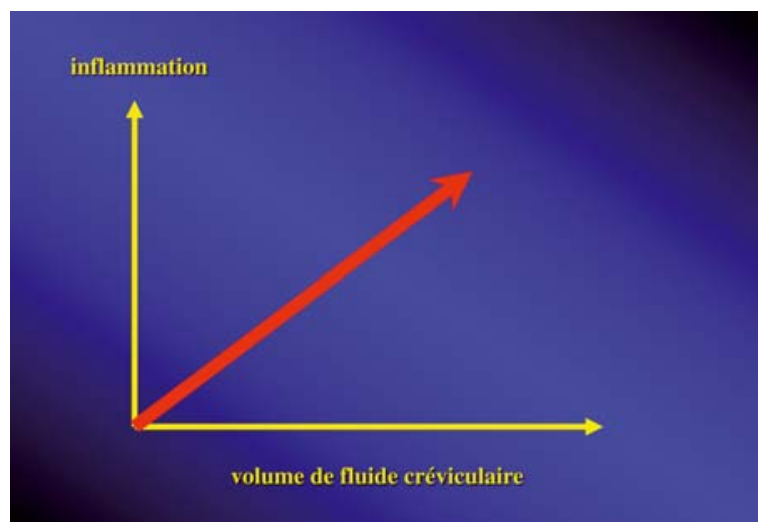

\section{HISTOLOGIE DU SILLON GINGIVO-IMPLANTAIRE}

Dès 1974, James, à partir d'une expérimentation sur le singe, montre autour de l'implant léquivalent d'un épithélium de jonction, sans cellules inflammatoires. Son étude histochimique par la technique acide de Schiff (PAS) révèle une grande ressemblance avec l'attache épithéliale dentaire : lépithélium de jonction produit «des mucopolysaccharides avec des propriétés capables de créer une adhésion avec la surface de l'implant». Depuis, de nombreux auteurs ont retrouvé léquivalent d'une lame basale et d'hémidesmosomes entre des implants en titane et l'épithélium environnant - Schroeder et al. [3], Hansson et al. [4], Gould et al. [5] qui concluent : "les résultats montrent que les cellules épithéliales sáttachent à la surface en titane par le moyen d'une lame basale et d'hémidesmosomes, pour beaucoup de la même façon que lattache épithéliale est appliquée à la surface de la dent».

Lorigine de cette attache apparaissant sur une surface gingivale exempte de dents a été étudiée dès 1981 par Swoope et James [6], plus récemment identifiée par Mackenzie et Tonetti [7] qui concluent, après une étude sur les anticorps monoclonaux spécifiques des cytokératines et de l'ICAM-1 (marqueurs des trois phénotypes de l'épithélium gingival), examinés en

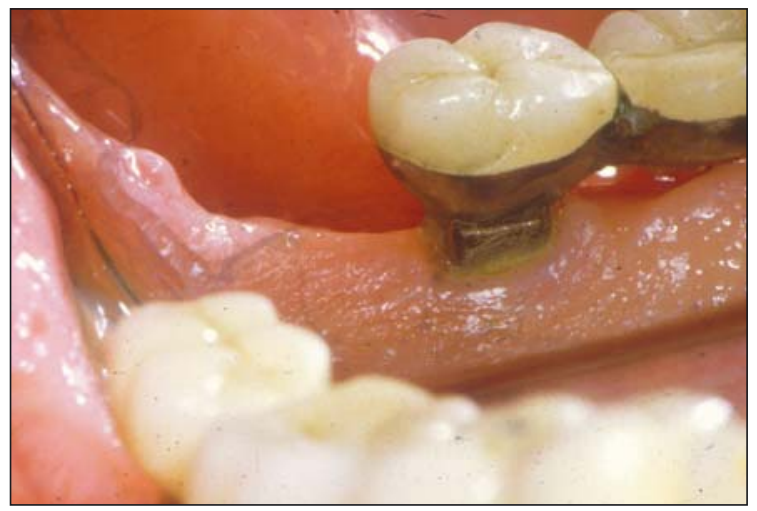

$\triangle$ Fig. 4 :

Le site de prélèvement. La gencive est de couleur rose et présente un piqueté en peau d'orange, caractéristiques d'une gencive saine. $\triangleleft$ Fig. 3 :

Il existe une corrélation

directe entre l'augmentation

de la sécrétion de fluide

créviculaire et l'augmentation

de P'inflammation du sillon

gingivo-dentaire (d'après

Oliver [2]).

immunofluorescence : "...les observations montrent la formation d'épithéliums oral, sulculaire et jonctionnel qui étaient indifférentiables, sur le plan phénotypique, de ceux de lépithélium naturel. Ce degré de reprogrammation de l'expression génique épithéliale est une observation surprenante».

Au niveau du tissu conjonctif, Arnold et al. [8] et Hansson et al. [4] ont montré qu'au contact du titane, la cellule dominante était le fibroblaste, qu'il existait des fibres de collagène avec quelques lymphocytes et quelques macrophages, cellules de défense toujours retrouvées dans ces structures très sollicitées. Sur un modèle expérimental sur le chien, Buser et al. [9] ont montré qu'il existait des fibres perpendiculaires directement apposées sur l'implant, vues à $70 \%$ dans les sites kératinisés et à $7 \%$ dans les sites non kératinisés. Plus récemment, Nevins et al. [10] ont apporté la preuve histologique d'une attache conjonctive à un implant dentaire chez l'homme: "L'attache conjonctive supra-crestale est évidente à la vue de coupes colorées au bleu de toluidine/AZUT en microscopie électronique, et en regardant les coupes sous lumière polarisée pour que l'orientation oblique des fibres soit confirmée par la suite au $M E B »$ ».

Un prélèvement réalisé sur un implant de type lame en place depuis quinze ans [11], avec une coloration topographique comme le trichrome de Masson, confirme la réalité d'une structure très proche de l'attache épithélio-conjonctive dentaire (fig. 4 à 10).

La coloration histochimique à l'acide périodique de Schiff (PAS), réalisée sur le même échantillon et révélant la présence (PAS+) ou l'absence (PAS-) de mucopolysaccharide, responsables de l'adhésion des cellules épithéliales sur un substrat, permet de confirmer que l'attache épithélio-conjonctive implantaire est semblable en tout point à l'attache épithélio-conjonctive dentaire (fig. 11 à 14).

$\mathrm{Au}$ niveau de sa physiologie, depuis les études initiales de Ponitz et al. [12] et de Mac Kinney et al. [13], de nombreux auteurs ont montré qu'il existait une sécrétion créviculaire autour d'un implant, comparable à celle retrouvée autour d'une dent et subissant une augmentation identique de sa sécrétion en cas d'inflammation. 


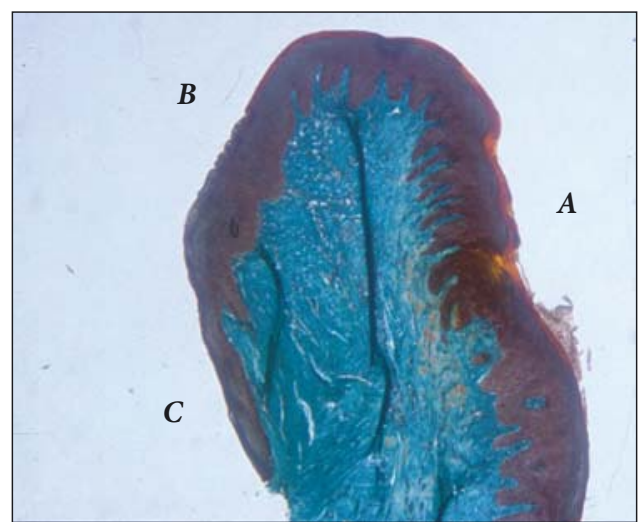

$\triangle$ Fig. 5 :

La coloration au trichrome de Masson montre 3 zones distinctes : lépithélium externe (A), la zone sulculaire (B) et la zone en contact avec l'implant (C), ressemblant à un long épithélium de jonction.

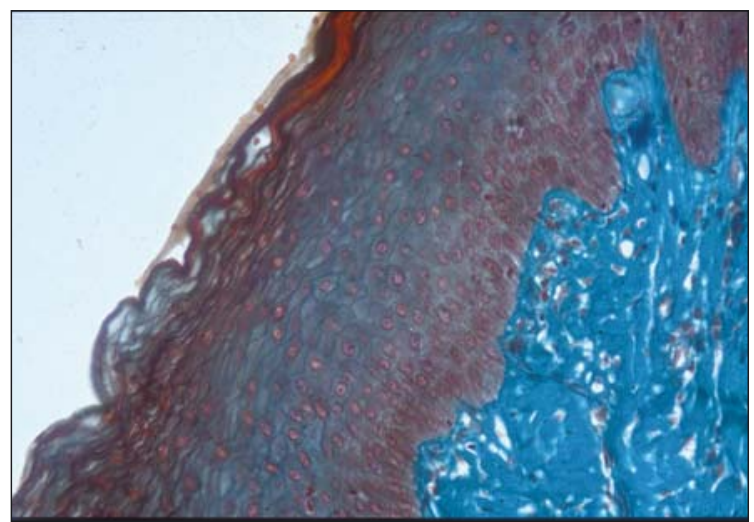

$\triangle$ Fig. 7 :

Au niveau sulculaire, la kératine a disparu, de même que les digitations. L'épithélium présente cependant un grand nombre de couches cellulaires. Il n'existe aucune cellule inflammatoire.

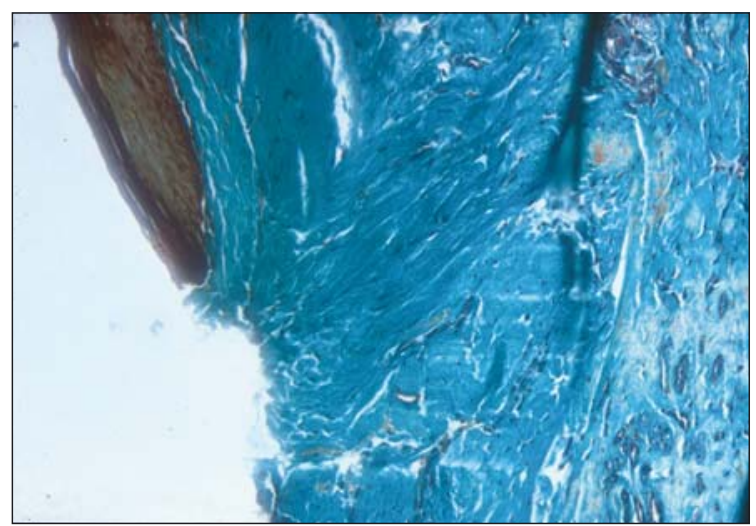

$\triangle$ Fig. 9

À la base de l'épithélium, le tissu conjonctif présente une orientation de ses fibres perpendiculaire à la surface de l'implant, caractéristique d'une attache conjonctive.

Au niveau microbiologique, les études initiales de l'équipe de Brånemark [14] ont montré que l'on retrouvait sur le site des bactéries non pathogènes (Cocci G+, bâtonnets non mobiles), et que les bactéries G- et les spirochètes ne sont que rarement retrouvés, avec des infiltrats inflammatoires très

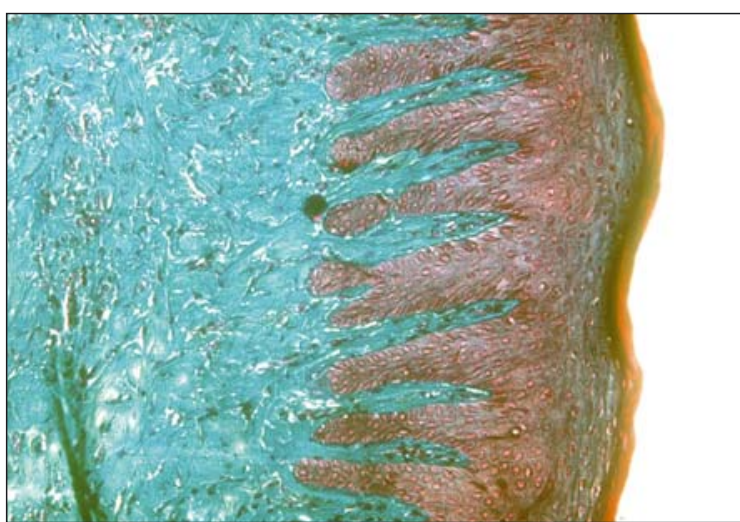

$\triangle$ Fig. 6 :

Lépithélium externe est kératinisé et présente des digitations dans le tissu conjonctif.

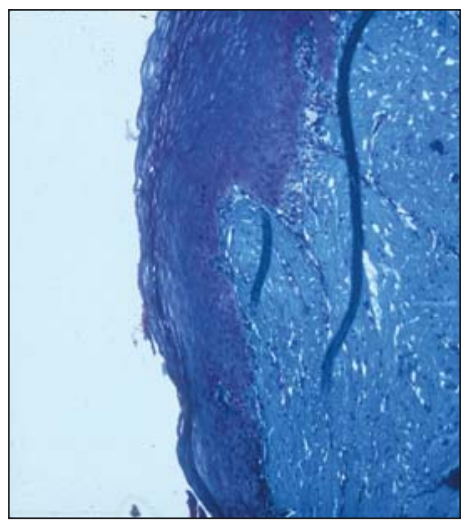

$\triangle$ Fig. 8 :

Au contact de l'implant, le nombre de couches cellulaires va en diminuant.

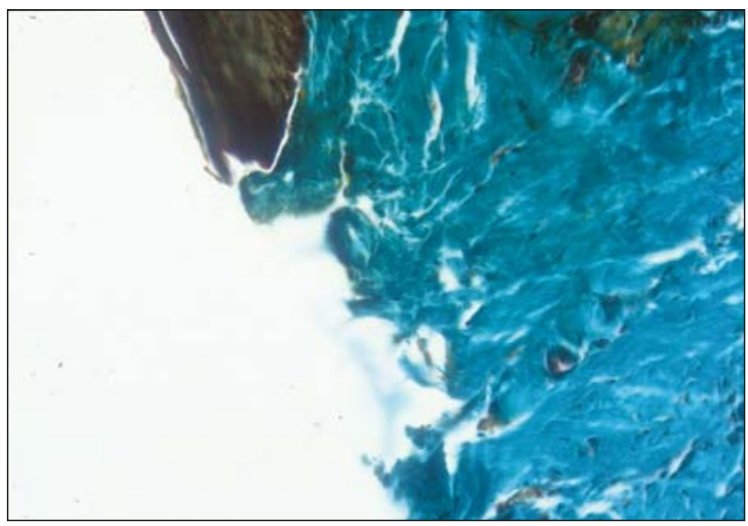

$\triangle$ Fig. 10

Un plus fort grossissement confirme l'orientation perpendiculaire des fibres de collagène.

limités en nombre et en surface.

La ressemblance du sillon gingivo-implantaire avec le sillon gingivo-dentaire laisse supposer qu'il sera affecté par les mêmes pathologies, avec des images histologiques semblables, ce qui sera développé dans un prochain article. 


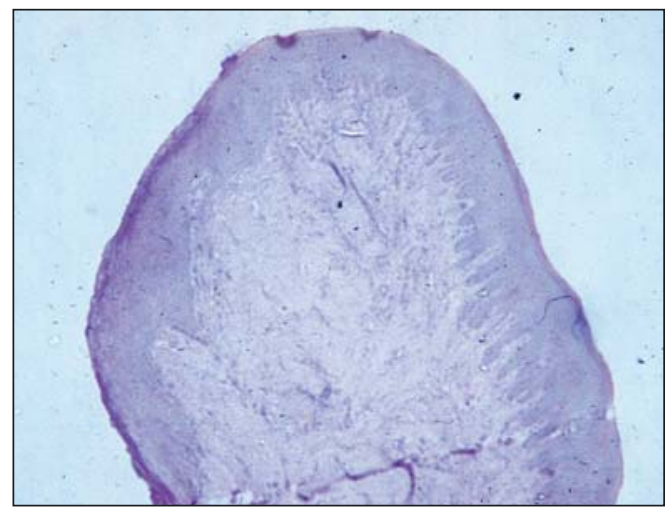

$\triangle$ Fig. 11 :

Le même échantillon est coloré à l'acide périodique de Schiff qui révèle la présence de mucopolysaccharide (violet foncé).

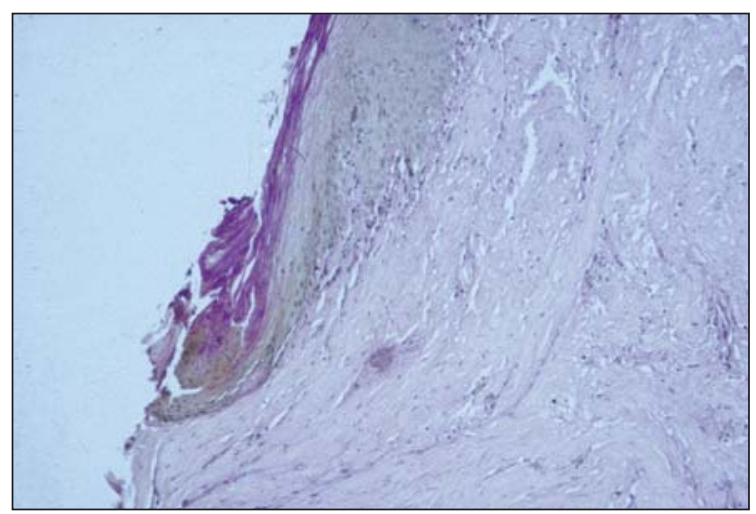

$\triangle$ Fig. 13:

La zone épithéliale à la base du sillon est fortement réactive à l'acide périodique de Schiff et ne présente plus que quelques couches cellulaires.

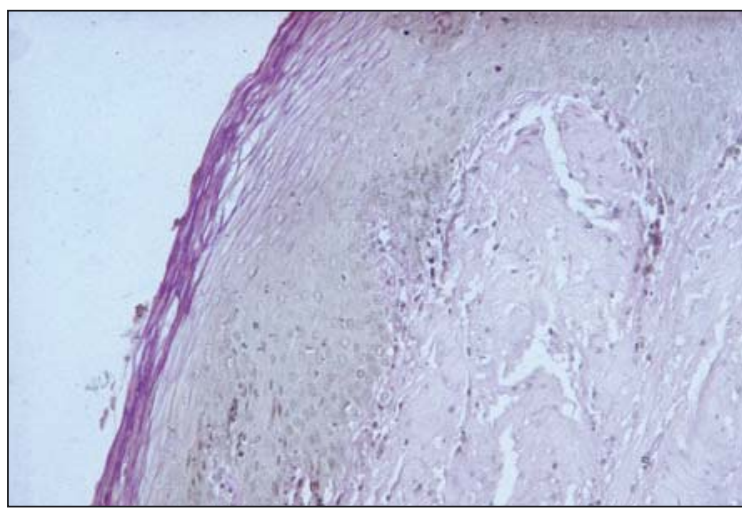

$\triangle$ Fig. 12 :

Lépithélium au contact de l'implant est fortement réactif à l'acide périodique, traduisant la présence de mucoplysaccharide, donc d'un mécanisme d'adhésion des cellules épithéliales sur le titane par l'intermédiaire d'hémidesmosomes.

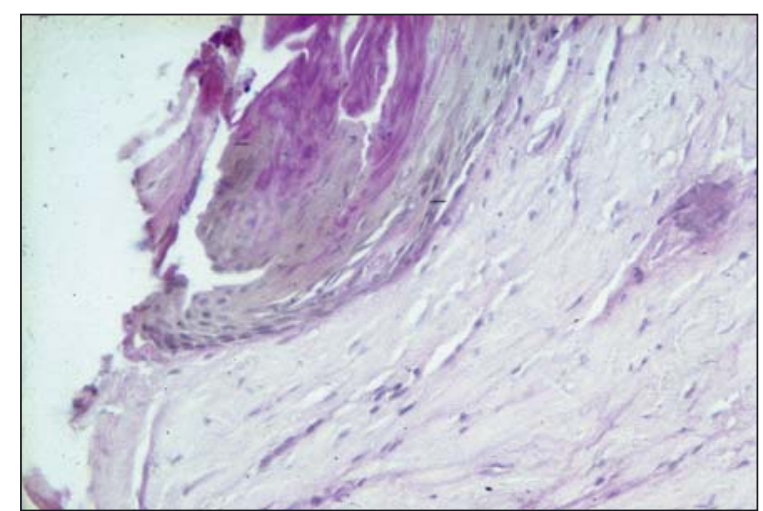

$\triangle$ Fig. 14 :

Un plus fort grossissement confirme la réaction très positive à l'acide, ainsi que l'orientation perpendiculaire des fibres conjonctives situées directement sous lépithélium, caractéristiques d'un ancrage au niveau du titane.

\section{Bibliographie}

[1] Cimasoni G. The crevicular fluid. Bâle : Karger édit, 1974.

[2] Oliver MC. The correlation between clinical scoring, exsudate measurements and microscope evaluation of the inflammation in the gingiva. J Periodont Periodontics 1969;40:201-209.

[3] Schroeder A, Zypen E, Sutter F. The reactions of bone, connective tissue and epithelium to endosteal implant with titanium sprayed surface. J Maxillofac Surg 1981;9(1):15-25.

[4] Hansson PD, Albrektsson T, Brånemark PI. Structural aspect of the interface between tissue and titanium implant. J Prosthet Dent 1983; 50(1):108-113.

[5] Gould RI, Brunette DM, Westbury L. The attachment mechnism of epithelial cells to titanium in vitro. J Periodont Res 1983;16:611-619.
[6] Swoope EM, James RA. A longitudinal study of hemi-desmosomes formation on the dental implant-tissue interface. Oral Implantology 1981;9(3): 412-425.

[7] Mackenzie I.C., Tonetti M.S. F ormation of normal gingival epithelial phenotypes around osseo-integrated oral implants in humans. J Periodontol, 1995,66:933-943.

[8] Arnold L.F., Huard T.K., Baram P. Cultivation of periodontal ligament fibroblasts on dental implant material. J Dent Res 1974;53(6):1368-1385.

[9] Buser D., Krekeler G., Schröder A. Fiber orientation in the peri-implant mucosa in an experimental model in dog. Z Zahnärztl Implantol 1989;5:15-28.

[10] Nevins M., Nevins M.L., Camelo M., Boyesen J.L., Kim D.M. Preuve histologique d'une attache conjonctive à un implant dentaire chez l'homme. Parodont Dent Rest 2008;28:111-121.

[11] Bert M., Missika P. Les implants ostéointégrables. CdP édit, Paris, 1992.

[12] Ponitz D.P., Gershkoff A., Wells H. Passage of orally administered tetracycline into the gingival crevice around natural teeth and dental implants. Dent Clin N Amer, 1970,14,1:125-142.

[13] Mac Kinney R.V., Koth D.I. The singlecrystal sapphire endosteal dental implants. Material characteristics and 18-month experimental trials. J Prosthet Dent, 1982,55:96-100.

[14] Lekholm U., Ericsson I., Adell R., Slotts $J$. The condition of the soft tissues at the toothand fixture abutments supporting fixed bridges. A microbiological and histological study. J Clin Periodont, 1986,13:58-69. 